\title{
MEJORAS EN LOS PROCESOS FORMATIVOS: PERSPECTIVAS EN LA UNIVERSIDAD CATÓLICA DE VALPARAÍSO
}

Gladys Jiménez* 


\section{RESUMEN}

Recrear un nuevo humanismo desde su visión y misión es el desafío de la Universidad. Los procesos docentes han de procurar, para los estudiantes, una formación sólida en principios; el desarrollo de habilidades y competencias requeridas en la sociedad del conocimiento; el trabajo interdisciplinario, la calidad docente, la conformación de comunidades de aprendizaje y la incorporación apropiada de metodologías interactivas y de nuevas tecnologías de la información, fomentando el compromiso con las personas, el diálogo, la confianza recíproca y la participación en el quehacer universitario. Tal proceso puede impulsarse y fortalecerse por medio de tres líneas de trabajo: formación valórica de los jóvenes, mejoramiento de la calidad de los procesos formativos y autoevaluación y acreditación de carreras.

\section{ABSTRACT}

The challenge faced by this University is to recreate a new type of humanism from its own viewpoint and in accordance with its mission. The goal of teaching processes should be to have students achieve a sound basis in terms of principles, develop their skills and the competencies needed in a knowledge-based society. This needs to be accompanied by interdisciplinary work, good teaching quality and the formation of learning communities and the adequate use of interactive methodologies and new information technologies to encourage commitment to people, dialogue, mutual trust and participation in university activities. A process such as this can be fostered and promoted by three lines of work: developing values among young people, improving the quality of teaching processes and by self-assessment and accreditation of degree programs. 


\section{MEJORAS EN LOS PROCESOS FORMATIVOS: PERSPECTIVAS EN LA UNIVERSIDAD CATÓLICA DE VALPARAÍSO}

\section{ORIENTACIONES ESTRATÉGICAS EN LA FORMACIÓN DE PREGRADO}

Desde su fundación, en marzo de 1928, la Universidad Católica de Valparaíso nace para servir a los jóvenes definiendo en su visión la formación de personas integrales, caracterizadas por el sello valórico institucional, competentes para un desempeño profesional prestigiado, en constante actualización, comprometidas socialmente y capaces para asumir tareas en diferentes ámbitos y culturas ${ }^{1}$.

Para ello, esta Universidad ha desarrollado una ininterrumpida labor académica de formación, de investigación y extensión, orientada hacia el "cultivo, a la luz de la fe, de las ciencias, las artes y las técnicas a través de la creación y comunicación del conocimiento, y la formación de graduados y profesionales con vocación de servicio a la sociedad, en el marco valórico del Magisterio de la Iglesia"2. Ha ido atesorando con sentido renovado una rica tradición y una presencia activa en el destino de la región y del país, siempre bajo el lema legado por sus fundadores: Fe y Trabajo.

Hoy, la definición de sus orientaciones estratégicas responde tanto a su identidad como a la consolidación de una forma de pensar y construir el presente y futuro de su quehacer.

En esa perspectiva, la Universidad Católica de Valparaíso ha ido dando nuevo sentido a su labor formativa, con una actitud siempre atenta a reconocer y analizar el futuro, sus requerimientos y desafíos. Sus acciones se nutren de su visión y de la realidad que se va presentando y que busca anticipar para responder con éxito al desafío formativo, tanto en lo valórico con un sello distintivo de nuestra Universidad, como en el desarrollo de habilidades y competencias

1. Orientaciones Estratégicas, Visión de la Universidad Católica de Valparaíso, 2000.

2. Ibidem. 
requeridas para el aprendizaje a lo largo de la vida. Formación general sólida junto con formación inicial en la especialidad, apropiada para las competencias académicas y profesionales definidas; mayor flexibilidad para los estudiantes y efectividad estructural e individual en los procesos respectivos, son la base de esta nueva mirada.

En la perspectiva de los lineamientos estratégicos, los procesos docentes han de procurar logros sustantivos en objetivos tales como: una formación de los estudiantes sólida en principios cristianos, de modo que impregnen el mundo con sus valores desde sus respectivos ejercicios profesionales; el desarrollo de habilidades y competencias requeridas en la sociedad del conocimiento; el trabajo interdisciplinario; la calidad docente; la conformación de comunidades de aprendizaje y la incorporación apropiada de metodologías interactivas y de nuevas tecnologías de la información. Asimismo, se fomentará el compromiso con las personas, la convivencia, el diálogo, la confianza recíproca y la participación en las distintas dimensiones del quehacer universitario.

\section{PANORAMA ACTUAL: LÍNEAS DE CAMBIO PRIORITARIAS IMPULSADAS}

De gran impacto en los diferentes ámbitos de la vida social han sido las transformaciones ocurridas en la sociedad desde las últimas décadas del siglo pasado. En este escenario, la Universidad Católica de Valparaíso se ha hecho cargo del sentido íntimo de tales cambios, teniendo en consideración tanto los lineamientos estratégicos sucintamente antes esbozados como la tarea de recrear un nuevo humanismo. Ha impulsado y fortalecido prioritariamente tres líneas de trabajo: una, vinculada a la formación valórica de los jóvenes, otra, centrada en el mejoramiento de la calidad de los procesos formativos y la tercera en la autoevaluación y acreditación de carreras.

\section{a) Formación valórica}

En el proceso educativo universitario está presente el desarrollo de habilidades profesionales, el cultivo de los talentos personales y la formación valórica de los educandos. Esta última adquiere hoy una especial relevancia. 
La formación valórica tiene en su centro a la persona del estudiante; su logro compromete transversalmente a todos los miembros de la comunidad universitaria y, por ende, a lo que acontece en las diferentes dimensiones de su quehacer. Esta dimensión trasciende la sala de clases y, en tanto proceso de formación, se desarrolla en la convivencia y en la cotidianeidad de la vida universitaria en general, así como en la particularidad del aula. Ello se logra a través del aprendizaje profesional y del rol testimonial que tienen los docentes como mediadores de este proceso; durante la participación en actividades académicas, deportivas, artísticas, culturales y solidarias; al interior de los grupos de estudio y de convivencia, y en los espacios extracurriculares donde el estudiante participa libremente, comparte y da lugar a sus iniciativas de diversa índole.

Es en esta diversidad de ámbitos y transversalidad de acciones que se fortalece la impronta valórica institucional de los procesos docentes formativos. Espacios de encuentro y diálogo, de expresión, canalización y autogestión de las propuestas estudiantiles a través de proyectos y de participación responsable en redes de distinta naturaleza, constituyen, entre otros niveles formales, una realidad propicia para que los estudiantes reafirmen su identidad, fortalezcan su sentido de pertenencia y asuman paulatinamente su proyecto de vida.

Para los jóvenes, lo preponderante de la formación valórica está en la vivencia del rigor y la alegría; en la experiencia directa de sentirse reconocidos en sus particularidades y fortalezas; respetados en su persona, derechos y dificultades; exigidos en sus deberes y responsabilidades; acogidos en sus proposiciones e invitaciones; integrados como miembros activos de la comunidad universitaria y mediante la experiencia de los vínculos interpersonales, en la receptividad para con sus puntos de vista, en el diálogo y en el acompañamiento a sus intereses, interrogantes y proyectos.

La estrategia de trabajo a través de la presentación de proyectos, que se ha ido afianzando con el tiempo, ha significado la materialización de una variedad de iniciativas en las que destacan, principalmente, la creatividad y la capacidad de gestión de los estudiantes, su vocación de servicio y el afán por el trabajo bien hecho. 
En esta misma línea, y teniendo en cuenta la diversidad de los intereses, expectativas y sus características, se desarrollan acciones formativas orientadas a consolidar redes con participación de profesores y estudiantes. Ellas contribuyen a la manifestación de actitudes positivas, principalmente de solidaridad, de respeto por el sentido de la convivencia, por la vocación de servicio, por la responsabilidad y por el comportamiento ético profesional y personal, así como por el uso recreativo del tiempo libre y los estilos de vida saludable.

Especial contribución en este aspecto corresponde al Programa de Bienvenida para los Alumnos de Primer Año, que imprime en los estudiantes recién ingresados una impronta institucional que los vincula con sus profesores, compañeros y con la disciplina elegida. En esta misma línea se desarrollan los proyectos "CONFIA", que enfatizan la capacidad de autogestión estudiantil, el trabajo en equipo y el compromiso con el logro de iniciativas solidarias, académicas, artísticas, culturales y recreativas.

Otras iniciativas que fortalecen el proceso formativo son las competencias deportivas interescuelas, cuya realización se da en un contexto valórico centrado en el respeto por el juego limpio y las normas de aceptación universal. La práctica del deporte, que responde a intereses personales, conduce al uso sano del tiempo libre, al desarrollo del sentido de pertenencia y a la superación personal en torno de propósitos comunes.

En esta perspectiva valórica son de gran relevancia los programas de prevención, que consideran la participación estudiantil en equipos interdisciplinarios orientados a la superación de conductas desadaptativas y a la promoción de factores protectores, privilegiando, como bien social, estilos de vida saludable, personales y comunitarios.

En este mismo plano de iniciativas que convocan a profesores y estudiantes, cabe mencionar los proyectos de tutoría académica, orientados al apoyo del proceso formativo de los alumnos en los ámbitos del desarrollo vocacional y personal, para descubrir y valorar los talentos personales, fomentar actitudes positivas frente al estudio y las habilidades cognitivas y sociales, propias de las exigencias de la formación profesional vinculadas al " aprender a aprender". 
De la misma forma, se lleva a cabo un nutrido programa de charlas, cursos y talleres, que permitan potenciar factores protectores entre nuestros estudiantes y fortalecer acciones que apoyen la superación de crisis vitales.

Especial contribución a la formación valórica le corresponde al Programa de Estudios Generales, que tiene por finalidad contribuir a la formación de profesionales cultos, capaces de desenvolverse en otras áreas mediante el contacto con campos del saber ajenos a su propia disciplina, haciéndoles partícipes de la diversidad de estudios cultivados en esta Universidad. De esta forma, se amplía el horizonte del proceso formativo a través de una visión del mundo complementaria a la mirada de la disciplina específica, contribuyendo así a la formación integral del alumno.

En la Universidad Católica de Valparaíso la formación valórica sucede tanto en el ejercicio de la docencia-fundamentalmente a través de lo testimonial-como en los espacios y momentos extracurriculares de la Universidad, jugando un rol preponderante el intercambio que sucede natural, intencional y transversalmente en la experiencia de ser comunidad de personas.

\section{b) Calidad de los procesos formativos}

La Universidad integra en los procesos formativos tanto el desarrollo de competencias profesionales como de habilidades transversales en los planos humanos, interpersonales y comunicacionales, que permiten interpretar y discernir en un mundo globalizado, con nuevos requerimientos sociales y nuevos desempeños laborales. El cultivo de los dones recibidos; la valoración de la convivencia universitaria y del diálogo como su método; la consolidación del sentido de identidad, pertenencia, compromiso y autorregulación responsable, son aspectos que se potencian simultáneamente junto con las dimensiones propiamente académicas del proceso formativo.

Las tareas visualizadas en este contexto implican una mirada audaz, que debe integrar estrategias de desarrollo del pregrado y la concurrencia de elementos que hemos enunciado precedentemente y que ahora enumeramos: profesores comprometidos con el proceso formativo de los estudiantes y con la calidad docente; condiciones apropiadas para el estudio y la convivencia; docencia interactiva y adecuado uso de la tecnología de la información; la inter- 
disciplinariedad y la promoción de un cambio organizacional asociado a la búsqueda de mayor flexibilidad, desarrollo curricular en colaboración y a la formación de comunidades de aprendizaje.

Con esta perspectiva, se promueven acciones en diversos ámbitos: capacitación en didáctica universitaria, innovación en la docencia, investigación en pedagogía universitaria, autoevaluación y evaluación, dirigidas al mejoramiento de la calidad de los procesos formativos.

La primera de las acciones, que se orienta a dar nuevo significado a la labor docente poniendo especial énfasis en la tarea de la enseñanza y el aprendizaje, pretende construir un proceso de desarrollo para el profesor universitario y los ayudantes, vinculado a actividades de mejoramiento de la práctica docente en las dimensiones curricular, metodológica y de evaluación del aprendizaje de los estudiantes, comprendiendo los aspectos actitudinales y valóricos propios del quehacer educativo. Se fortalecen de esta manera: el sentido de la formación en contraste con el concepto de información; la capacidad para crear entornos de aprendizaje acordes con el desarrollo de estrategias metacognitivas; capacidades de autoaprendizaje de los estudiantes y el uso de los avances tecnológicos.

La segunda de las acciones en este plano se refiere a la innovación. Esta impulsa la implementación y el intercambio de experiencias exitosas de los profesores, referidas a proyectos de innovación en docencia interactiva, avanzando en una progresiva participación en la virtualidad como apoyo a la docencia presencial y en un sostenido mejoramiento de los espacios docentes que favorecen las condiciones para el estudio y la convivencia.

La tercera, investigación en pedagogía universitaria, pretende impulsar la indagación en este ámbito, como una estrategia de mejoramiento e innovación del trabajo docente propiamente tal, y de los aspectos afines al trabajo formativo de los estudiantes y las prácticas pedagógicas académicas.

\section{c) Autoevaluación y acreditación de carreras}

Por último, están las acciones en materia de evaluación, proceso consustancial al mejoramiento de la calidad de la formación y de sus 
resultados. En esta perspectiva, se avanza en la valoración de los procesos de evaluación y autoevaluación, particularmente vinculadas al sentido de retroinformación y de dar cuenta de resultados, de modo de reconocer y superar dificultades, así como de consolidar fortalezas y tomar decisiones fundadas en evidencias de la propia realidad. Es en este marco que se intenta incrementar las acciones de evaluación en el nivel de aula, de los procesos docentes, de los resultados de tales procesos y de los distintos servicios ofrecidos, en una perspectiva de mejoramiento cualitativo de la docencia. Asimismo, la participación gradual en acciones de autoevaluación y acreditación de carreras de pregrado, quehacer orientado por la Comisión de Evaluación Institucional, permite identificar fortalezas y debilidades en las carreras evaluadas y, por ende, tomar decisiones sustantivas para mejorar y consolidar la calidad y pertinencia de los programas ofrecidos.

\section{LAS ACCIONES FUTURAS: IDENTIDAD EDUCATIVA, EL DESAFÍO DE LA FORMACIÓN INTEGRAL Y LOS PROCESOS E INSTANCIAS DE ASEGURAMIENTO DE LA CALIDAD DE LA DOCENCIA}

Las universidades han sido reconocidas históricamente como impulsoras del conocimiento en las diferentes épocas por las que les ha tocado transitar. Hoy, para muchos, son instituciones que enfrentan nuevos desafíos y exigencias que cuestionan su misión tradicional.

En este plano, se aprecia, por un lado, una tendencia hacia la especialización y fragmentación creciente del conocimiento. Al interior de las aulas, los alumnos se encuentran con saberes inabarcables y diversos junto con dificultades para poder establecer las relaciones necesarias entre ellos y su integración al saber profesional contextualizado. A esta dificultad, se suman tendencias conducentes a una concepción meramente acumulativa de los conocimientos, cuya dimensión se ahonda cuando se tiene la certidumbre de su acelerada caducidad.

Es necesario, entonces, avanzar en la tarea de diseñar estructuras curriculares innovadoras y flexibles, en la diversificación de la oferta de programas en función de las necesidades de la sociedad y en la 
vinculación de los estudios de pregrado con los de postgrado. En términos más específicos, el carácter constructivo del conocimiento nos desafía a identificar criterios de selección de las materias que componen los currículos; a vincular los procesos de enseñanza y aprendizaje de la docencia con la investigación académica; a implementar metodologías que formen a los estudiantes en la autonomía y que releven la capacidad de aprender que es más importante que lo aprendido.

Los docentes, en consonancia con la revolución de las comunicaciones, deberán independizarse de su posición como fuente única del conocimiento, para dar sentido y orientación al conocimiento que está libremente disponible para los alumnos.

Los estudiantes, a su vez, deberán ser capaces de interpretar la realidad; de dialogar en forma interdisciplinaria con otros profesionales, de tal modo que puedan mirar esta realidad desde distintas perspectivas; de aprender a ser críticos, a construir significados y a otorgar sentido y singularidad a las propias acciones para tomar decisiones adecuadas al contexto laboral en que se desempeñen.

Así, pues, los estudiantes se verán enfrentados al hecho de aprender a pensar, mientras que los profesores tendrán que ser capaces de implementar estrategias didácticas que conduzcan a la reflexión, a la metacognición y al aprendizaje estratégico, que motiven a trabajar en equipo y a integrar redes organizacionales de modo de aprender a colaborar con sus semejantes. Es decir, los docentes deben potenciar sus habilidades sociales y competencias académicas para comunicar, explicar y argumentar su saber y quehacer; como asimismo, escuchar, apreciar las opiniones de los demás y comprender los distintos puntos de vista para interpretar apropiadamente la realidad y sus circunstancias.

Los nuevos desafíos del siglo XXI y las exigencias derivadas de la globalización, revitalizan la apertura y extensión de los espacios universitarios hacia la comunidad regional, nacional e internacional. La debida vinculación entre la formación teórica y la formación contextualizada en la realidad, permite consolidar la construcción del conocimiento en una perspectiva social, propiciando oportunidades 
que potencian la capacidad para resolver problemas y retroalimentar los procesos de formación personal y profesional. En este sentido, nuestro deber es establecer condiciones que permitan a nuestros egresados incorporarse adecuadamente a un escenario globalizado, dinámico y cambiante. Tal desafío implica velar por la interdisciplinariedad y por el cultivo de la capacidad para dar respuestas integrales a los problemas del hombre y del mundo actual e incorporar definitivamente metodologías de autoevaluación y evaluación de los procesos docentes, tanto en los aprendizajes de los estudiantes como en las estrategias utilizadas para su logro.

Responder tanto a la formación integral de sus estudiantes como al aseguramiento de la calidad de los procesos formativos significa, para la Universidad Católica de Valparaíso, situar la tarea de articular adecuadamente los procesos de personalización de cada estudiante con su formación profesional desde los valores humanistas y cristianos; reconocer la disponibilidad del saber; colocar de relieve el sentido de la vida, con especial énfasis y cuidado en el respeto por el ser humano, cualquiera sea su condición, y practicar las virtudes básicas de la convivencia, la valoración del diálogo y el sentido de identidad, pertenencia y compromiso social. Tareas éstas, sin lugar a dudas, cautivantes. 